\title{
Chronic inflammation and lung fibrosis: pleotropic syndromes but limited distinct phenotypes
}

\author{
AH Gifford ${ }^{1,6}$, M Matsuoka $^{1,6}$, LY Ghoda $^{2,3}$, RJ Homer $^{4}$ and RI Enelow ${ }^{1,5}$
}

Experimental models of lung fibrosis have been disappointing in predicting therapeutic responses to a wide variety of interventions in clinical fibrosing lung diseases. There are multiple potential reasons, but this fundamentally calls into question the validity of the models and their fidelity to clinical syndromes. We propose that the clinical diseases associated with pulmonary fibrosis, although manifesting a broad array of widely different clinical presentations and features, result in essentially two distinct phenotypes of fibrosis that we will describe. The most common and problematic of these are not effectively modeled experimentally. In this review, we present several clinical entities as examples of the phenotypic distinctions. The first two represent the extremes: postinflammatory fibrosis observed in hypersensitivity pneumonitis (HP) and dysregulated matrix deposition as observed in idiopathic pulmonary fibrosis (IPF). We also present a third clinical entity, that of lung disease associated with rheumatoid arthritis (rheumatoid lung), representing a condition that can manifest as either phenotype, and offering a potential opportunity to explore the mechanisms underlying the pathogenesis of the two distinct fibrotic phenotypes.

\section{INTRODUCTION}

Common to nearly all types of lung fibrosis is some form of "injury" to the lung, whether immune mediated or not, although exceptions of course exist (such as asbestosis and silicosis). Nevertheless, there are themes and mediators common to all forms of fibrosis. Histopathologic descriptors are used in clinical practice, such as "usual interstitial pneumonia (UIP)," which represents a very specific descriptive pattern of abnormalities associated with entities such as "idiopathic pulmonary fibrosis (IPF)," but may also describe one form of rheumatoid lung disease. Although the clinical circumstances in which this pattern is observed may differ greatly, the nature of the fibrotic process appears quite similar, including the resistance to therapeutic intervention, the relentless progression, and abysmal prognosis.

Other histopathologic descriptors are less specific, and may reflect a varied spectrum of patterns of inflammation and secondary fibrotic deposition. Descriptors in current use include such entities as nonspecific interstitial pneumonitis (NSIP) with or without fibrosis, and these types of patterns are often observed in conditions in which the fibrotic processes may remit with successful anti-inflammatory therapy. This pattern, which we refer to as "wound healing," is observed in any number of diseases that may be more responsive to therapy, such as many of the autoimmune lung diseases (e.g., systemic lupus erythematosis), and interestingly is also observed in some types of rheumatoid lung. Regardless of the underlying disease, this pattern of inflammation and fibrosis behaves in a fundamentally distinct fashion than UIP, and several extreme examples of these phenotypes will be outlined below in order to emphasize the differences.

\section{HYPERSENSITIVITY PNEUMONITIS: A PARADIGMATIC EXAMPLE OF FIBROSIS AS POSTINFLAMMATORY "WOUND HEALING"}

There are a number of forms of "fibrotic lung disease" in which inflammation of the lung is clearly a precursor to the development of fibrosis, such as hypersensitivity pneumonitis

\footnotetext{
${ }^{1}$ Department of Medicine, Geisel School of Medicine at Dartmouth, Lebanon, New Hampshire, USA. ²Department of Neurology, Beckman Research Institute, City of Hope Medical Research Center, Duarte, California, USA. ${ }^{3}$ Keren Pharmaceuticals, San Mateo, California, USA. ${ }^{4}$ Department of Pathology and Laboratory Medicine, Yale University School of Medicine, New Haven, Connecticut, and VA Connecticut Healthcare System, West Haven, Connecticut, USA. ${ }^{5}$ Department of Microbiology/ Immunology, Geisel School of Medicine at Dartmouth, Lebanon, New Hampshire, USA. ${ }^{6}$ These authors contributed equally to this work. Correspondence: RI Enelow (Richard.i.enelow@dartmouth.edu) 
(HP). HP is an interstitial inflammatory disease characterized by a vigorous lymphocytic infiltration of the lung parenchyma provoked by repeated inhalation of environmental antigens, the growing list of which is reviewed in detail elsewhere. ${ }^{1}$ Farmer's lung and bird fancier's lung are prototypic diseases representing this syndrome. The spectrum of clinical presentation varies and acute, subacute, and chronic forms have been described. The chronic form of HP may progress to extensive pulmonary fibrosis if responsible antigens are not identified and continued exposure occurs, although this tends to be unusual.

Abundant numbers of inflammatory cells are observed in the lung tissue and bronchoalveolar lavage fluid of these patients, and anti-inflammatory therapies are usually very effective. When steroids are given early in the course of the disease, the inflammation usually resolves, and the postinflammatory fibrosis generally does not progress. The patterns of gene expression profiles are also very different between the postinflammatory fibrosis observed in HP and the dysregulated matrix deposition, observed in IPF (discussed below). Strikingly distinct gene expression profile patterns were found by Selman et al. ${ }^{2}$ in lung biopsy specimens from HP and IPF patients. HP lungs showed evidence of a robust inflammatory component, including many genes associated with infiltrating activated $\mathrm{T}$ cells, as well as interferon- $\gamma$-inducible chemokines. In contrast, IPF lungs were characterized by the expression of genes associated with tissue remodeling, epithelial, and fibroblast genes. (Similarly, the gene expression profile of another inflammatory disease, pulmonary sarcoidosis with fibrosis, was examined and found to be enriched for immune response genes, similar to the gene sets unregulated in HP, but decidedly distinct from those observed in samples from IPF. ${ }^{3}$ )

The immunology and pathogenesis of HP has been long studied, yet remains poorly understood. Early studies focused on the role of specific immunoglobulin Gs (precipitins) and formation of immune complexes in the lungs, although this is controversial. ${ }^{4}$ Both mouse and human HP studies found increased $\mathrm{T}$ helper type 1 (Th1) cytokines in the lungs, such as interferon- $\gamma$, interleukin (IL)-12, IL-18, and tumor necrosis factor- $\alpha$, supporting involvement of Th1 CD $4+$ and CD $8+\mathrm{T}$ cells in the pathogenesis. ${ }^{5-7}$ Although numerous studies have suggested a relationship between Th2-mediated inflammation and fibrosis, in contrast to Th1-mediated inflammation (reviewed in $\mathrm{Wynn}^{8}$ ), this is a false dichotomy, as both experimental and clinical literature do not convincingly support this notion. Indeed, the gene expression patterns in clinical HP demonstrate evidence of type 1 inflammation; ${ }^{2}$ however, one of the paradigmatic Th2 cytokines, IL-13, has been clearly associated with fibrotic processes, although this effect is probably independent from its association with type 2 inflammatory responses, as suggested by the phenotype of experimental fibrosis associated with IL-13 overexpression in mice (Lee et al., ${ }^{9}$ see preclinical models below).

\section{IPF: FIBROSIS AS AN EXAMPLE OF UNREMITTING DYSREGULATED MATRIX DEPOSITION}

IPF represents the opposite extreme of the spectrum of clinical fibrotic entities, compared with those associated with postinflammatory fibrosis. It has gradually become apparent that the histopathological finding of inflammation, which is common (even if minimal), is entirely incidental to the otherwise relentless dysregulated deposition of extracellular matrix, in a manner that is always progressive and ultimately fatal. No form of treatment has been shown to be effective in halting this process, although many clinical trials have been performed and more are underway. There are abundant etiopathologic theories, some based upon rare but informative clinical phenotypes. ${ }^{10}$ Gene-expression profiles of human specimens ${ }^{11}$ suggests a combination of injury (acute, multiple, or chronic) to the lung in a genetically susceptible individual predisposed to inadequate repair mechanism. ${ }^{12}$ Such individuals may be prone to premature epithelial cell senescence or depletion of the epithelial stem cell niche, and lung injury (of uncertain nature) may result in unsuccessful "attempts" at repair of damaged epithelium, leading to disorganized and unremitting fibrosis, destruction of the alveolar architecture, and loss of the gas exchange units. ${ }^{13}$

The hallmark of any fibrotic process is the deposition of extracellular matrix materials. The amount and composition as well as the degree of organization of this matrix are all profoundly important in maintenance of elasticity, the integrity of the alveolar-capillary interface, and ventilation/perfusion matching. ${ }^{14-16}$ IPF represents an extreme circumstance of disorganized matrix deposition. Transforming growth factor- $\beta$ (TGF- $\beta$ ) has been implicated as a downstream mediator of fibrosis in not only IPF, but also in most other fibrotic syndromes. ${ }^{17}$ Increased levels of TGF- $\beta$ have been found in both bronchoalveolar lavage fluid and lung tissue derived from IPF patients. ${ }^{16,18-20}$ It is also likely a major driving force in the process of epithelial-to-mesenchymal transition (EMT) in the lung. ${ }^{21,22}$ EMT is a fundamental process in early development where cells take on a mesenchymal phenotype during the formation of the mesoderm and induction of the neural crest. The demonstration of an increase in Twist1, a transcription factor that plays the role of master regulator of these processes, is consistent with the suggestive role of EMT in IPF. $^{23,24}$ The contribution of EMT to IPF, however, is an active topic of debate. ${ }^{25,26}$ Regardless, the contribution of TGF- $\beta$ in fibrotic lung disease is not in question-it is both a target and an inducer of Twist 1 and Twist 1 is regulated through a feed-forward mechanism contributed both by Twist 1 itself and nuclear factor- $\kappa \mathrm{B}$, induced by inflammatory signals. ${ }^{27}$ Indeed, as Twist 1 has been implicated in maintenance of the stem-like state, aberrant expression of this developmental transcription factor is also consistent with fibrocytes or mesenchymal stem cells playing a role in disorganized fibrotic states. ${ }^{28-31}$

\section{RHEUMATOID ARTHRITIS-ASSOCIATED INTERSTITIAL LUNG DISEASE: A UNIQUE OPPORTUNITY TO UNDERSTAND THE PATHOGENESIS OF BOTH PHENOTYPES}

Rheumatoid arthritis (RA) lung disease can take many forms, although the two paradigmatic entities are: (i) an inflammatory cellular interstitial pneumonitis (NSIP), treatable with anti-inflammatory agents, and (ii) a phenotype largely indistinguishable from that of untreatable dysregulated matrix deposition (UIP). There is little question that RA is an inflammatory 
disease, and treatment with anti-inflammatory agents is effective at suppressing the disease manifestations, although it is a common observation that with the UIP form of rheumatoid lung, aggressive anti-inflammatory therapy has little, if any, impact on the progressive matrix deposition in the lung, despite complete suppression of the arthritis. Multiple other autoimmune syndromes that often involve the lung may manifest either pulmonary phenotype, and there is no evidence that one is a precursor to the other.

RA is the most prevalent autoimmune disease, affecting an estimated $0.6-1.0 \%$ of adults worldwide. ${ }^{32-35}$ Radiologic investigations have identified myriad abnormalities in the small airways and lung parenchyma of $42-63 \%$ of RA patients depending on diagnostic modalities and criteria applied to these populations. ${ }^{36-39}$ Pulmonary involvement in RA confers a mortality ratio of 2.86 compared with having RA without lung disease. ${ }^{40}$ That RA, a discrete, well-defined rheumatologic (inflammatory) disease, can manifest as either of two distinct patterns of interstitial lung disease (UIP or NSIP) highlights the unique opportunity for understanding the nature of the pathways and factors that may fundamentally alter the fibrotic phenotype rendering it clinically autonomous of the inflammatory process.

Interestingly, tobacco smoke inhalation may increase the likelihood that UIP, rather than NSIP, develops in RA patients. Limited data suggest that a history of smoking is more common among RA patients whose lung biopsy specimens display UIP as opposed to NSIP or other patterns ${ }^{41,42}$ Reasons for this predilection among smokers are unclear. Cigarette smoke extract depletes intracellular glutathione, increases lipid peroxidation, and induces necrosis in human alveolar epithelial cells in a dosedependent manner, ${ }^{43}$ suggesting that oxidative stress is a significant trigger for type I pneumocyte loss and alveolar disintegrity. These alveolar wall defects can be repaired by differentiation of type II pneumocytes stimulated by growth factors like hepatocyte growth factor and keratinocyte growth factor elaborated by proximate fibroblasts. ${ }^{44}$ Type II pneumocytes typically display some degree of resistance to noxious insult, perhaps by inducible antioxidant enzymes, ${ }^{45}$ but tobacco smoke may be particularly harmful to these cells critical for affective alveolar repair. Generally, the toxic, necrotic, or proapoptotic effect of tobacco smoke may contribute to cell death of all epithelial populations, including type II alveolar cells that proliferate in response to type I cell injury, and thereby contribute to the disorganized repair observed in the IPF lung. ${ }^{46}$ Smoking could also limit alveolar repair at the level of progenitor differentiation. Pneumocytes believed to be in transition from the type II to type I phenotype based on biomarker alteration undergo apoptosis and necrosis more readily after cigarette smoke extract exposure than differentiated type II pneumocytes. ${ }^{47}$ It remains unclear whether type II pneumocytes (or other epithelial stem-like progenitor cells, e.g., p63 + distal alveolar stem cells ${ }^{48}$ ) in the RA lung are uniquely susceptible to tobacco smoke, whether this contributes to premature senescence of this population, and whether this leads to UIP. (It is well documented that smoking is an independent risk factor for $\mathrm{IPF}^{49}$ ) An interesting hypothesis that might be worthy of exploration is the underappreciated link between chronic inflammation and mesenchymal stem cell aging, ${ }^{50}$ and this may represent an informative link between the aberrant wound healing seen in UIP associated with RA and "lone UIP" (IPF) in which stem cell senescence via noninflammatory mechanisms may be a contributory factor. ${ }^{51,52}$

\section{PRECLINICAL MODELS}

Although much has been learned about the mediators of normal wound healing using experimental models such as bleomycininduced lung injury, ${ }^{53}$ the limitations of the model suggest that further insights will require experimental systems that more closely mimic the various phenotypic features unique to the clinical syndromes of dysregulated matrix deposition. IL-13, a prototypic Th2 cytokine, has been extensively studied. Lee et al. ${ }^{9}$ created an inducible lung-specific IL-13 transgenic mouse line that exhibited a variety of abnormalities resembling human allergic airway disease but also developed progressive fibrosis in the airway and lung parenchyma. The profibrotic effect of IL-13 appears to involve, not unexpectedly, TGF- $\beta$, potentially produced by profibrotic macrophages (of the "M2" phenotype ${ }^{54,55}$ ), but these mechanisms are not well characterized (reviewed by $\mathrm{Wynn}^{8}$ ). After injury, lung epithelium undergoes repair and/or regeneration to repopulate the lost epithelium and resurface the area resulting in transient "fibrosis," which resolves with time. Bleomycin induces an acute lung injury with an inflammatory response, which offers an excellent model of postinflammatory wound healing, and the preclinical literature is replete with examples of "abrogation of fibrosis" with anti-inflammatory interventions in this model. ${ }^{56} \mathrm{It}$ is unfortunate, but not unexpected, that successful preclinical candidates fail in clinical trials as the fidelity of the models to clinically relevant conditions is questionable.

The fibrotic phase of the murine system is probably better modeled by overexpression of TGF- $\beta,{ }^{57}$ the prototypic molecule that (along with its family members) appears to drive expression of extracellular matrix genes in both normal and aberrant wound healing. This model, although limited itself in the depth of fidelity to clinical disease, has been informative with respect to the dependence of fibrosis on epithelial cell apoptosis, which is observed in the clinical syndromes of dysregulated matrix deposition. ${ }^{46}$ Other models in which epithelial susceptibility to apoptosis is manipulated also provide insight into this aspect of the problem. ${ }^{54}$ Conversion of latent to active TGF- $\beta$ in the lung requires the action of the lung-specific $\alpha(\mathrm{v}) \beta 6$ integrin, and its targeted deletion demonstrates the dependence of fibrosis in bleomycin-induced injury, even in the presence of significant injury and inflammation. ${ }^{58}$ Gene expression analysis clearly demonstrates two distinct sets of genes induced in the bleomycin model, one entirely inflammatory, and the other associated with wound healing. ${ }^{59}$ That said, there was limited overlap with the gene expression patterns observed in samples of lung from patients with IPF. ${ }^{2}$ Other interesting models exist that may inform specific aspects of the clinical conditions, such as a model of spontaneous arthritis with chronic interstitial pneumonitis, the full expression of which requires environmental insults. ${ }^{60,61}$ It would be of considerable interest to further investigate such a 
model, and interposing genetic or other interventions informed by studies of human cells/tissues in fibrotic disease states (e.g., defects in the $\mathrm{WNT} / \beta$-catenin pathway ${ }^{62}$ ). This type of iterative refinement of experimental models may ultimately be a more advantageous strategy than continued "lateral exploration" of individual models.

\section{CONCLUSION}

The notion that all forms of fibrosing lung disease can be reduced to two distinct phenotypes obviously represents a gross oversimplication of the variety of complex diseases and the varying nature of lung fibrosis. Nevertheless, it may be a useful starting point in the generation of more useful preclinical models. The basic premise that pulmonary fibrosis is essentially a unitary process may be a reflection of the state of current preclinical research (reductionist by nature), but is thoroughly undermined by clinical evidence. This suggests that significant reconsideration be given to the value of the evidence gained from presently available animal models. Furthermore, the opportunities for understanding the factors that drive the development of relentless deposition of extracellular matrix in the most common (and most severe) types of fibrotic disease may reside in the study of patients with the most common autoimmune disease, RA, where $\sim 50 \%$ of the patients develop pulmonary abnormalities. Incremental insights gained from well-designed patientoriented studies may inform the development of more useful animal models where rational identification of therapeutic targets can be translated into candidates for evaluation in future clinical trials. The information gained from clinical observations (often in unexpected fields ${ }^{51,52}$ ) should also be integrated into the design and use of preclinical models to develop more refined hypotheses and experimentation. Insights into the pathogenesis and treatment of these aggressive, debilitating, and fatal diseases may require a concerted bedside to bench to bedside approach to have a positive impact on the hitherto abysmal 20 -year record of clinical trials.

\section{ACKNOWLEDGMENTS}

We gratefully acknowledge the support of PHS grants R01-AI069360, U19-AI083024 (RIE), and P20-RR01878 (AHG); we like gratefully acknowledge the generous support of Dartmouth SYNERGY (Center for Clinical and Translational Research). This article is dedicated to the memory of John A Kelly, MD, an important intellectual contributor to the conceptual basis of this review.

\section{DISCLOSURE}

LYG is a co-founder and shareholder of Keren Pharmaceuticals, a privately held biotechnology company developing a proprietary gene silencing technology for therapeutic applications. The other authors declared no conflict of interest.

\section{(C) 2012 Society for Mucosal Immunology}

\section{REFERENCES}

1. Costabel, U., Bonella, F. \& Guzman, J. Chronic hypersensitivity pneumonitis. Clin. Chest Med. 33, 151-163 (2012).

2. Selman, M. et al. Gene expression profiles distinguish idiopathic pulmonary fibrosis from hypersensitivity pneumonitis. Am. J. Respir. Crit. Care Med. 173, 188-198 (2006).
3. Lockstone, H.E. et al. Gene set analysis of lung samples provides insight into pathogenesis of progressive, fibrotic pulmonary sarcoidosis. Am. J. Respir. Crit. Care Med. 181, 1367-1375 (2010).

4. Salvaggio, J.E. \& Robert, A. Cooke memorial lecture. Hypersensitivity pneumonitis. J. Allergy Clin. Immunol. 79, 558-571 (1987).

5. Ye, Q., Nakamura, S., Sarria, R., Costabel, U. \& Guzman, J. Interleukin 12 , interleukin 18 , and tumor necrosis factor alpha release by alveolar macrophages: acute and chronic hypersensitivity pneumonitis. Ann. Allergy Asthma Immunol. 102, 149-154 (2009).

6. Mroz, R.M., Korniluk, M., Stasiak-Barmuta, A. \& Chyczewska, E. Upregulation of Th1 cytokine profile in bronchoalveolar lavage fluid of patients with hypersensitivity pneumonitis. J. Physiol. Pharmacol. 59 (Suppl 6), 499-505 (2008).

7. Mitaka, K. et al. Th2-biased immune responses are important in a murine model of chronic hypersensitivity pneumonitis. Int. Arch. Allergy Immunol. 154, 264-274 (2011).

8. Wynn, T.A. Integrating mechanisms of pulmonary fibrosis. J. Exp. Med. 208, 1339-1350 (2011).

9. Lee, C.G. et al. Interleukin-13 induces tissue fibrosis by selectively stimulating and activating transforming growth factor beta(1). J. Exp. Med. 194, 809-821 (2001).

10. Garcia, C.K. Idiopathic pulmonary fibrosis: update on genetic discoveries. Proc. Am. Thorac. Soc. 8, 158-162 (2011).

11. Kaminski, N. \& Rosas, I.O. Gene expression profiling as a window into idiopathic pulmonary fibrosis pathogenesis: can we identify the right target genes? Proc. Am. Thorac. Soc. 3, 339-344 (2006).

12. Selman, M., Pardo, A. \& Kaminski, N. Idiopathic pulmonary fibrosis: aberrant recapitulation of developmental programs? PLoS Med. 5, e62 (2008).

13. Leslie, K.O. Idiopathic pulmonary fibrosis may be a disease of recurrent, tractional injury to the periphery of the aging lung: a unifying hypothesis regarding etiology and pathogenesis. Arch. Pathol. Lab. Med. 136, 591-600 (2012).

14. Rozin, G.F., Gomes, M.M., Parra, E.R., Kairalla, R.A., de Carvalho, C.R. \& Capelozzi, V.L. Collagen and elastic system in the remodelling process of major types of idiopathic interstitial pneumonias (IIP). Histopathology 46, 413-421 (2005).

15. Schneider, D.J. et al. Cadherin-11 contributes to pulmonary fibrosis: potential role in TGF-beta production and epithelial to mesenchymal transition. FASEB J. 26, 503-512 (2012).

16. Hiwatari, N., Shimura, S., Yamauchi, K., Nara, M., Hida, W. \& Shirato, K. Significance of elevated procollagen-III-peptide and transforming growth factor-beta levels of bronchoalveolar lavage fluids from idiopathic pulmonary fibrosis patients. Tohoku J. Exp. Med. 181, 285-295 (1997).

17. Garcia-Sanchez, O., Lopez-Hernandez, F.J. \& Lopez-Novoa, J.M. An integrative view on the role of TGF-beta in the progressive tubular deletion associated with chronic kidney disease. Kidney Int. 77, 950-955 (2010).

18. Lomas, N.J., Watts, K.L., Akram, K.M., Forsyth, N.R. \& Spiteri, M.A. Idiopathic pulmonary fibrosis: immunohistochemical analysis provides fresh insights into lung tissue remodelling with implications for novel prognostic markers. Int. J. Clin. Exp. Pathol. 5, 58-71 (2012).

19. Khalil, N. et al. Regulation of the effects of TGF-beta 1 by activation of latent TGF-beta 1 and differential expression of TGF-beta receptors (T beta R-I and T beta R-II) in idiopathic pulmonary fibrosis. Thorax 56 , 907-915 (2001).

20. Khalil, N., O'Connor, R., Gold, L.I., Parekh, T. \& Raghu, G. Biological effects of transforming growth factor-beta(1) in idiopathic pulmonary fibrosis may be regulated by the activation of latent transforming growth factor-beta(1) and the differential expression of transforming growth factor-beta receptors. Chest 120 (1 Suppl), 48S (2001).

21. Coward, W.R., Saini, G. \& Jenkins, G. The pathogenesis of idiopathic pulmonary fibrosis. Ther. Adv. Respir. Dis. 4, 367-388 (2010).

22. Kasai, H., Allen, J.T., Mason, R.M., Kamimura, T. \& Zhang, Z. TGF-beta1 induces human alveolar epithelial to mesenchymal cell transition (EMT). Respir. Res. 6, 56 (2005).

23. Pozharskaya, V. et al. Twist: a regulator of epithelial-mesenchymal transition in lung fibrosis. PLoS One 4, e7559 (2009).

24. Bridges, R.S., Kass, D., Loh, K., Glackin, C., Borczuk, A.C. \& Greenberg, S. Gene expression profiling of pulmonary fibrosis identifies Twist1 as an antiapoptotic molecular "rectifier" of growth factor signaling. Am. J. Pathol. 175, 2351-2361 (2009). 
25. Morbini, P., Inghilleri, S., Campo, I., Oggionni, T., Zorzetto, M. \& Luisetti, M. Incomplete expression of epithelial-mesenchymal transition markers in idiopathic pulmonary fibrosis. Pathol. Res. Pract. 207, 559-567 (2011).

26. Rock, J.R. et al. Multiple stromal populations contribute to pulmonary fibrosis without evidence for epithelial to mesenchymal transition. Proc. Natl. Acad. Sci. USA 108, E1475-E1483 (2011).

27. Pham, C.G. et al. Upregulation of Twist-1 by NF-kappaB blocks cytotoxicity induced by chemotherapeutic drugs. Mol. Cell. Biol. 27, 3920-3935 (2007).

28. Andersson-Sjoland, A., Nihlberg, K., Eriksson, L., Bjermer, L. \& Westergren-Thorsson, G. Fibrocytes and the tissue niche in lung repair. Respir. Res. 12, 76 (2011).

29. Antoniou, K.M. et al. Investigation of bone marrow mesenchymal stem cells (BM MSCs) involvement in Idiopathic Pulmonary Fibrosis (IPF). Respir. Med. 104, 1535-1542 (2010).

30. Cakouros, D., Raices, R.M., Gronthos, S. \& Glackin, C.A. Twist-ing cell fate: mechanistic insights into the role of twist in lineage specification/ differentiation and tumorigenesis. J. Cell. Biochem. 110, 1288-1298 (2010).

31. Isenmann, S. et al. TWIST family of basic helix-loop-helix transcription factors mediate human mesenchymal stem cell growth and commitment. Stem Cells 27, 2457-2468 (2009).

32. Helmick, C.G. et al. Estimates of the prevalence of arthritis and other rheumatic conditions in the United States. Part I. Arthritis Rheum. 58, 15-25 (2008).

33. Englund, M., Joud, A., Geborek, P., Felson, D.T., Jacobsson, L.T. \& Petersson, I.F. Prevalence and incidence of rheumatoid arthritis in southern Sweden 2008 and their relation to prescribed biologics. Rheumatology (Oxford, England) 49, 1563-1569 (2010).

34. Scublinsky, D. et al. The prevalence of rheumatoid arthritis in Argentina: a capture-recapture study in a city of Buenos Aires province. J. Clin. Rheumatol. 16, 317-321 (2010).

35. Anagnostopoulos, I. et al. The prevalence of rheumatic diseases in central Greece: a population survey. BMC Musculoskelet. Disord. 11, 98 (2010).

36. Gochuico, B.R. et al. Progressive preclinical interstitial lung disease in rheumatoid arthritis. Arch. Intern. Med. 168, 159-166 (2008).

37. Kanat, F., Levendoglu, F. \& Teke, T. Radiological and functional assessment of pulmonary involvement in the rheumatoid arthritis patients. Rheumatol. Int. 27, 459-466 (2007).

38. Mohd Noor, N., Mohd Shahrir, M.S., Shahid, M.S., Abdul Manap, R., Shahizon Azura, A.M. \& Azhar Shah, S. Clinical and high resolution computed tomography characteristics of patients with rheumatoid arthritis lung disease. Int. J. Rheum. Dis. 12, 136-144 (2009).

39. Mori, S., Cho, I., Koga, Y. \& Sugimoto, M. Comparison of pulmonary abnormalities on high-resolution computed tomography in patients with early versus longstanding rheumatoid arthritis. J. Rheumatol. 35, 1513-1521 (2008).

40. Bongartz, T. et al. Incidence and mortality of interstitial lung disease in rheumatoid arthritis: a population-based study. Arthritis Rheum. 62, 1583-1591 (2010).

41. Lee, H.K. et al. Histopathologic pattern and clinical features of rheumatoid arthritis-associated interstitial lung disease. Chest 127, 2019-2027 (2005).

42. Tsuchiya, Y. et al. Lung diseases directly associated with rheumatoid arthritis and their relationship to outcome. Eur. Respir. J. 37, 1411-1417 (2011).
43. Kode, A., Yang, S.R. \& Rahman, I. Differential effects of cigarette smoke on oxidative stress and proinflammatory cytokine release in primary human airway epithelial cells and in a variety of transformed alveolar epithelial cells. Respir. Res. 7, 132 (2006).

44. Fang, K.C. Mesenchymal regulation of alveolar repair in pulmonary fibrosis. Am. J. Respir. Cell. Mol. Biol. 23, 142-145 (2000).

45. Bhandari, V., Maulik, N. \& Kresch, M. Hyperoxia causes an increase in antioxidant enzyme activity in adult and fetal rat type II pneumocytes. Lung 178, 53-60 (2000).

46. Barbas-Filho, J.V., Ferreira, M.A., Sesso, A., Kairalla, R.A., Carvalho, C.R. \& Capelozzi, V.L. Evidence of type II pneumocyte apoptosis in the pathogenesis of idiopathic pulmonary fibrosis (IFP)/usual interstitial pneumonia (UIP). J. Clin. Pathol. 54, 132-138 (2001).

47. Kosmider, B., Messier, E.M., Chu, H.W. \& Mason, R.J. Human alveolar epithelial cell injury induced by cigarette smoke. PLoS One 6, e26059 (2011).

48. Kumar, P.A. et al. Distal airway stem cells yield alveoli in vitro and during lung regeneration following H1N1 influenza infection. Cell 147, 525-538 (2011).

49. Raghu, G. et al. An official ATS/ERS/JRS/ALAT statement: idiopathic pulmonary fibrosis: evidence-based guidelines for diagnosis and management. Am. J. Respir. Crit. Care Med. 183, 788-824 (2011).

50. Lepperdinger, G. Inflammation and mesenchymal stem cell aging. Curr. Opin. Immunol. 23, 518-524 (2011).

51. Vece, T.J. et al. Rapid and progressive pulmonary fibrosis in 2 families with DNA repair deficiencies of undetermined etiology. J. Pediatr. 160, 700-702 e3 (2012).

52. Diaz de Leon, A. et al. Telomere lengths, pulmonary fibrosis and telomerase (TERT) mutations. PLoS One 5, e10680 (2010).

53. Moore, B.B. \& Hogaboam, C.M. Murine models of pulmonary fibrosis. Am. J. Physiol. Lung. Cell. Mol. Physiol. 294, L152-L160 (2008).

54. Homer, R.J., Elias, J.A., Lee, C.G. \& Herzog, E. Modern concepts on the role of inflammation in pulmonary fibrosis. Arch. Pathol. Lab. Med. 135, 780-788 (2011).

55. Murray, L.A. et al. TGF-beta driven lung fibrosis is macrophage dependent and blocked by Serum amyloid P. Int. J. Biochem. Cell Biol. 43, 154-162 (2011).

56. Wynn, T.A. Cellular and molecular mechanisms of fibrosis. J. Pathol. 214, 199-210 (2008).

57. Lee, C.G. et al. Early growth response gene 1-mediated apoptosis is essential for transforming growth factor beta1-induced pulmonary fibrosis. J. Exp. Med. 200, 377-389 (2004).

58. Munger, J.S. et al. The integrin alpha $v$ beta 6 binds and activates latent TGF beta 1: a mechanism for regulating pulmonary inflammation and fibrosis. Cell 96, 319-328 (1999).

59. Kaminski, N. et al. Global analysis of gene expression in pulmonary fibrosis reveals distinct programs regulating lung inflammation and fibrosis. Proc. Natl. Acad. Sci. USA 97, 1778-1783 (2000).

60. Keith, R.C. et al. A novel model of rheumatoid arthritis-associated interstitial lung disease in SKG mice. Exp. Lung Res. 38, 55-66 (2012).

61. Wakasa-Morimoto, C. et al. Arthritis and pneumonitis produced by the same $\mathrm{T}$ cell clones from mice with spontaneous autoimmune arthritis. Int. Immunol. 20, 1331-1342 (2008).

62. Vuga, L.J. et al. WNT5A is a regulator of fibroblast proliferation and resistance to apoptosis. Am. J. Respir. Cell Mol. Biol. 41, 583-589 (2009). 